Original article

https://www.journal-imab-bg.org

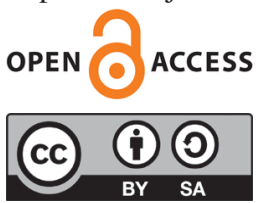

\title{
TREATMENT OF PROBLEMATIC SKIN WOUNDS BASED ON THE PLATELET-RICH PLASMA METHOD. OUR OWN ALGORITHMS FOR APPLI- CATION.
}

\author{
Tsvetan Sokolov ${ }^{1}$, Aneliya Manukova ${ }^{2}$, Vihar Kovachev ${ }^{3}$, Mancho Kovachev ${ }^{3}$ \\ 1) Orthopedics and traumatology clinic, UMBAL Kanev, Ruse, Bulgaria. \\ 2) Department of Electronics, University of Ruse, Ruse, Bulgaria. \\ 3) Clinic of Orthopedics and Traumatology, Medical Faculty, Medical Univer- \\ sity Pleven, Bulgaria.
}

\section{ABSTRACT}

OBJECTIVE: The objective of this paper is to present the application of our own algorithms for prevention and treatment of problematic skin wounds (PSW) by using the platelet-rich plasma (PRP) based on the first study on PRP application carried out in Bulgaria.

MATERIAL AND METHODS: The study was carried out at the Clinic of Orthopedics and Traumatology, UMBAL Kanev Ruse, for a period of 84 months - from February 2009 to September 2016. A total of 83 patients with PSW have been treated with platelet-rich plasma. Scores introduced by Cancela AM are used for the assessment of the respective wound. Each of these scores is used for assessing specific wound parameters.

RESULTS: Our own algorithms for prevention and treatment of PSW by PRP increase the percentage of successfully cured wounds. Prevention algorithm of applying PRP ensures that a high percentage of acute skin wounds will not turn into PSW. The proposed algorithms for prevention and treatment of PSW by applying PRP are an effective and safe way to reduce the uncured complicated skin wounds and ensure the subsequent normal life of patients. They also ensure more predictable skin healing.

CONCLUSION: Our own algorithms for prevention and treatment of PSW by PRP increase the percentage of successfully cured wounds. The proposed algorithms for prevention and treatment of PSW by applying PRP are an effective and safe way to reduce the uncured complicated skin wounds and ensure the subsequent normal life of patients.

Keywords: problematic skin wounds, platelet-rich plasma, algorithms for prevention and treatment

Problematic skin wounds (PSW) are a global medical and financial issue because patients usually have various concomitant diseases such as chronic venous insufficiency (CVI), diabetes mellitus, decubital skin defects, etc. [1, 2]. Approximately 100000 patients in Bulgaria have similar ulcerations [3].

PSW are defined as wounds that fail heal for at least 6 weeks. This category includes wounds resulting from high-energy injuries with bone or tendon prominence or metal surgical implant, as well as wounds where reconstructive surgery is not possible [4].

We view at PSW as an accumulation of acute (potentially problematic), hard-to-heal and chronic skin wounds. A potentially problematic wound is any wound that could turn into chronic. They are usually caused by a traumatic agent or surgical intervention in combination with concomitant metabolic or vascular diseases, and prolonged compression. Apart from etiological factors, the anatomical location, presence of infectious agents and patient's age also have a significant impact. In general, acute skin wounds primarily heal following the normal tissue regeneration pathway, but when accompanied by the above intrinsic and extrinsic factors they could become potentially problematic and subsequently chronic [5]. Lindholm et al. support the same view by proving that approximately $15 \%$ of acute wounds turn into chronic [6].

It is known that the major cause for impaired and delayed wound regeneration in PSW is the lack of growth factors in the damaged area, which adversely affects normal skin healing [7]. There various non-surgical (conservative) and surgical procedures for treatment and they lead to different outcome [8]. Consequently, the PRP method has been developed as the only one delivering growth factors, ensuring biological treatment and complete tissue regeneration of PSW [9].

Based on our significant clinical expertise, we have come to the conclusion that the development of clear prevention and therapeutic algorithms is essential for the proper application of PRP and for improvement of results. They offer accurate, initial assessment of each wound and ensure consistent and competent PRP treatment.

The algorithms we have proposed would help and facilitate any practitioner in selecting adequate treatment for complete wound healing and ensuring the patient's subsequent normal life. 


\section{MATERIAL AND METHODS}

\subsection{Patients}

The research was carried out at the Clinic of Orthopedics and Traumatology, UMHAT Kanev, Ruse for a period of 84 months - from February 2009 to September 2016. Out of a total of 83 patients with PSW have been treated with PRP. They have been studied, treated and monitored for at least 6 months. This representative statistical sample forms the first longitudinal research in the world on treating patients with PSW by applying PRP according to available data.

The distribution of patients by age has been as follows: $20,48 \%$ of them in the age group 70-79 years), $21,69 \%$ of them in the age group 50-59 years, $28,92 \%$ of them in the age group 60-69 years. The greatest share of patients with PSW - 71,09\%, fall into age groups over 50 years. Women and men with PSW were 42 males, 50,6\% and 41 females $49,4 \%$.

The distribution of patients by wound type shows the equal distribution in the three groups - acute wounds - 36,1\%, 30 patients; hard-to-heal wounds - 30,1\%, 25 patients and chronic wounds $-33,7 \%, 28$ patients.

Distribution of traumatic wounds, decubital wounds and wounds of inflammatory origin was as follows: 66 traumatic wounds $(79,5 \%) ; 15$ decubital wounds $(18,1 \%)$ and 2 wounds of inflammatory origin $(2,4 \%)$.

With patients have been isolated the following infectious agents: S.aureus -17 patients and E.coli -10 , K1.pneumoniae - 6, P.aeruginosa - 6, E.faecalis - 4. In 28 patients the following concomitant conditions have been observed: type 2 diabetes mellitus - 21 patients; CVI - 2 patients; phlebothrombosis of the shin and Parkinson's disease - 1 patient; paraparesis of lower extremities - 1 patient. The most common concomitant condition is type 2 diabetes mellitus (as the only concomitant disease) - a total of 21 patients $(75 \%)$.

\subsection{Analytical Method}

The treatment algorithm with PRP we used for PSW is as follows: first, there is surgical treatment and debridement of the wound. The first PRP application is on the second postoperative day. It is derived as follows: the necessary amount of blood is drawn in blood collection tubes containing 3,8\% sodium citrate. Tubes are centrifuged with a speed of $1800 \mathrm{rev} / \mathrm{min}$. for 8 minutes. Using pipettes the plasma is drawn off without leukocytes and $10 \%$ $\mathrm{CaCl}_{2}$ activator is added to it at a ratio of $1 \mathrm{ml}$. plasma to 50 microliters of activator or calcium gluconate at a ratio of $0,1 \mathrm{ml}$ activator to $1 \mathrm{ml}$. plasma. The results of the two activators according to our observations are identical. If fibrin clot with activated platelets is necessary according to our observation approx. $40 \mathrm{~min}$. should pass, in order to obtain the $3 \mathrm{D}$ gel. Activated plasma is used to infiltrate the wound edges and the fibrin clot is used to fill the wound itself. Sterile gauze dressing, soaked in $0,9 \%$ sodium chloride saline is made. The procedure should be repeated every week because activated platelets secrete growth factors within 7 days [10]. After removing the bandage the surrounding skin should be cleaned with povidone-iodine and the wound itself with saline solution. Subsequent manipulations after the initial wound processing and activated plasma application in a hospital might be performed in outpatient practice.

Wound assessment is done by the use of scores, introduced by Cancela et al. [11]. Specific wound parameters are presented numerically. Total Wound Score (TWS) stands for total wound parameters - oedema around the wound, erythema around the wound, puss discharge, fibrin, granulation, oedema at the bottom of the wound and ocher oedema. Total anatomic score (TAS) stands for total anatomic parameters- open bones and tendons, à.dorsalis pedis pulse and à.tibialis posterior pulse; Total Score of Wound data (TSWD) stands for the general wound conditions- size, depth, erosion and period of existence of the wound.

\subsection{Data Analysis}

The statistical analyses of our results have been processed by the SPSS software (Statistical Package for the Social Sciences) involving three nonparametric tests: Kolmogorov-Smirnov, Mann-Whitney and Wilcoxon to verify the significance of relations between features $[5,12]$. They are of crucial importance for the development of our treatment and prevention algorithms.

\subsection{Our algorithms}

Based on our in-depth experimental studies and analyses of treatment outcomes when applying PRP on PSW we have developed our own prevention and treatment algorithms for optimization of treatment for every single patient [5].

1.4.1 Algorithm for prevention application of platelet-rich plasma for treating potentially problematic skin wounds

Figure 1 shows the algorithm's structure in detail. It has been developed in order to minimize the risk of turning of an acute and potentially problematic skin wound into the chronic wound, by clear and precise assessment of indications and counter-indications and major risk factors.

The first step of the algorithm is diagnosing an acute, potentially PSW by taking a detailed history, assessing the local and somatic status and indications and counter-indications criteria. If the wound and the patient do not have the proper indications in accordance with Fig. 1 they have to be referred for any of the alternative treatment methods.

Patients with indications for PRP treatment should be checked against anatomical localization criteria. If the wound is located on the lower limb, Achilles tendon or calf, it is suitable for treatment with platelet-rich plasma. The rest of the cases are referred for treatment by standard methods depending on the pathology.

Next, the wound must be tested for infectious agents. If the test is negative the age criterion is applied. If an infectious agent is isolated it should be subjected 
to a microbiological test to determine its type. If it is S.aureus and E.coli we proceed by applying the age criterion. If the infection is Kl. pneumoniae, Enterococcus faecalis or Ps. aeruginosa antibiotic treatment based on an antibiogram is administered and the age criterion is applied

The analysis of our study has shown that age is crucial for treatment due to physiological changes experienced by patients [5]. The threshold age is set to 50 years. If the patient belongs to the age group below 50 years, they should be referred for standard treatment methods for the pathology. If they belong to the age group above 50 years they should be tested for comorbidities. In the absence of comorbidities, patient should be referred for surgical treatment of the wound and debridement, followed by application of platelet-rich plasma as indicated. If there are comorbidities, the underlying condition should be controlled followed by the application of our method.

1.4.2 Algorithm for treatment application of platelet-rich plasma on problematic skin wounds

Our algorithm for PRP application has been developed in order treatment of patients with various PSW to be carried out following strict rules for effective treatment leading to full tissue regeneration. It is applicable mostly for PSW referred by the prevention algorithm, for acute potentially problematic skin wounds, as well as for other hard-to-heal and chronic wounds. Figure 2 shows the algorithm's structure in detail.

For patients referred for PRP treatment, as described above, a detailed history should be taken and local and somatic status assessment should be performed again. At this stage wound parameters and wound, localization should be determined. The criteria specified should be used.

After determining the specific wound parameters follows thorough surgical debridement, second wound assessment and PRP application according to our method. Upon patient's weekly visits only cleansing the wound with saline solution PRP application is performed.

The wound healing process in time is assessed based on TWS, TAS and TSWD criteria. If the wound score is zero on the three criteria, treatment is successful and the problematic skin wound is fully healed. If the wound score is not zero, the TWS, TAS and TSWD scores have to be compared to the previous ones. If scores tend to decrease, PRP treatment is continued to complete healing of the PSW. If no decrease in scores is observed for three months, PSW is considered not affected by the treatment, it should be discontinued, and other treatment methods should be considered.

\section{RESULTS}

The results obtained have been processed through a variance analysis of the variables containing information about the initial and final data from each series of the study. The objective has been to determine the basic numerical characteristics of the variables and to check their variation trends through comparative analysis.

By comparing "treatment outcome" to "weeks of treatment" we have found that the majority of patients have fully recovered between $8(34,94 \%)$ and $12(28,91 \%)$ weeks, which is $63,85 \%$ (53 patients). Of this group, $91,38 \%$ of patients have been successfully treated by the PRP method.

The used PRP method has led to the full recovery of $21(100 \%)$ patients with only one concomitant disease: type 2 diabetes mellitus. Patients with only one concomitant condition such as shin phlebothrombosis and Parkinson's disease, paraparesis of lower extremities have been successfully treated with PRP.

Patient distribution by sex is uniform and the PRP method leads to equally good results for both sexes, therefore the method is not influenced by patient's sex. Treatment outcome for all patients treated by applying PRP is successful for $93,9 \%$ of patients with traumatic wounds, $88,8 \%$ with decubital wounds and $100 \%$ with wounds of inflammatory origin.

By comparing the interaction of results of «treatment outcome» and «weeks of treatment» to the presence of «infectious agent» we have found that for patients with S.aureus treatment should be extended to 24 weeks. When E.coli is present, treatment should be extended to 16 weeks, in the case of Kl.pneumoniae - treatment should reach 20 weeks. According to the results, the type of agent significantly impacts treatment duration.

The most common concomitant disease is type 2 diabetes mellitus (as an only concomitant condition), which combined with any infectious agent prolongs treatment. PSW anatomical location strongly impacts treatment duration and areas such as shin, foot and Achilles tendon, are especially difficult due to their anatomical features. Healing rate of skin ulcerations is not determined by the PSW depth. The etiological group has a moderate impact on PSW treatment duration. This allows drawing the conclusion that a larger percentage of patients with decubital wounds will have longer treatment periods as compared to patients with traumatic wounds. Our results and the statistical study carried out proves that the PRP method gives significant results for PSW treatment despite aggravating factors such as causing agent, concomitant conditions, location and $92,77 \%$ of patients have experienced complete wound healing. Only $7,22 \%$ of patient treated based on our method have unsatisfying results due to advanced concomitant conditions [5].

\section{DISCUSSION}

The most important conclusion, derived from the results of our study, is that our PSW treatment and prevention algorithms through the PRP method increase the percentage of successfully cured wounds. This is due to the proper selection of patients by strict initial wound assessment, risk factors evaluation, prescription of followup therapy, and observing the necessary sequence of steps for proper implementation of the method leading to com- 
plete recovery. After getting acquainted in detail with the available literature, we have not found any similar algorithms for PSW prevention and treatment by PRP application.

Of the first 25 patients with PSW we have treated with PRP, we have registered full recovery of $76 \%$ of them and this result is in full compliance with studies by other authors [7,11]. It is known by world literature sources that PRP treatment has some shortcomings. They are mainly biological (such as the insufficient quantity of autologous PRP and necessity of its quick processing) and technical ones (possible platelet fragmentation and lysis, early release of growth factors and cytokines leading to compromising their bioactivity) etc. [13]. Along with these disadvantages we have reported some difficulties and errors in the selection of our first group of patients and the treatment sequence when using the PRP method. We have reported $24 \%$ of them failed to fully heal and we have reached the conclusion that in order to improve results it is necessary to reconsider and streamline our diagnostic and treatment plan. In order to solve these problems, we have developed our own prevention and treatment algorithms. With their application, we have increased successful results from $76 \%$ to $92,78 \%$ and reduced unsuccessful cases from $24 \%$ to $7,22 \%$ [5].

There are many literature sources that prove the positive outcome of PRP application for chronic skin wound healing [14]. Research papers on its application for the prevention and treatment of acute skin wounds are quite limited [15]. As we have already mentioned $15 \%$ of them turn into chronic wounds [6]. So we have included in our study acute potentially PSW because they require extra precision of PRP treatment approach. This is accomplished through the introduction of a number of criteria and a sequence of their application. We consider that this sequence could be implemented through a clear and precise algorithm.

According to our study, $71,09 \%$ of PSW patients are above 50 years of age, which is related to a natural change of their biological state and occurrence of various comorbidities [5]. This has been confirmed by a number of authors [16]. Thus, the algorithm is necessary to streamline indications, counter-indications and major risk factors, which would have a negative impact on the treatment process. Apart from the patient's age other important factors having an impact on wound healing are: anatomical localization, presence of infectious agent and comorbidities. We have shown that anatomical localization on the lower limb deeply impacts treatment duration [5]. This has been supported by VanDen Kerkhof et al. [17]. We have shown that tissue regeneration of PSW is not influenced by a wound's anatomical depth [5].

Another risk factor that has a strong impact on the healing process is the infectious agent since the absence of pathogenic microorganisms is of crucial importance for normal skin regeneration without any complications. Ac- cording to our experience and scientific research, no antibiotic treatment is required upon the presence of S.aureus and E.coli (due to the antimicrobic properties of PRP), while in the case of Kl. pneumoniae, E. faecalis or Ps. aeruginosa antibiotic treatment is required [18].

The most common concomitant disease in our series has been diabetes mellitus. In order for a PSW to heal in such a case, it is necessary to control the condition, apply our PRP method and our own algorithms. Thus, we have achieved $100 \%$ wound healing. This approach has been successful in patients with other single comorbidities (CVI, phlebothrombosis, Parkinson's disease, etc.) [5]. When analyzing literature research we have found out that our method of treatment and results excel what has been published by other authors [19].

Chronic wounds resulting from diabetes mellitus, CVI or long-term compression are also a major therapeutic challenge. Now, most treatment protocols recommend standard treatment methods for an initial period of 4 weeks, after which a reassessment has to be made in terms of its area reduction [20]. Based on our clinical experience we distance ourselves from these protocols and recommend basing treatment on our algorithms. It saves time and money.

Our own prevention and treatment algorithms are based on our significant clinical expertise, in-depth research and statistical analyses. We use the digitalized criteria of Cancela et al. for wound assessment [11]. They are the foundation of our treatment algorithm because they allow optimal initial wound assessment, successful weekly, ongoing follow-up of tissue regeneration of PSW and reporting the final outcome. Criteria allow us to group the results obtained. So we are able to predict treatment duration.

The final result from PRP application based on our method and prevention and treatment algorithms for PSW is $92,78 \%$ full tissue regeneration [5]. By reviewing available literature we have found out that it excels the results published by many authors [7]. We believe that our better results could be explained by the application of our own algorithms since the PRP method has been identical with us and other researchers.

Both algorithms we have developed are different. The prevention algorithm has a diagnostic focus on acute, potentially PSW. We use it to properly select specific patients and wounds for adequate treatment and for minimization of the risk of turning them into chronic wounds. The treatment algorithm follows a strict sequence of treatment steps for all PSW. In this way, we reduce the risk of possible mistakes and complications in the course of tissue skin regeneration. We would like to strongly emphasize that both algorithms are logically linked, sometimes overlapping and following one another enhancing diagnostics and treatment in order to reach the best clinical results. The presented algorithms are easily and effectively applied in outpatient and patient care. 
Fig. 1. Algorithm for preventive application of platelet-rich plasma on potentially problematic skin wounds

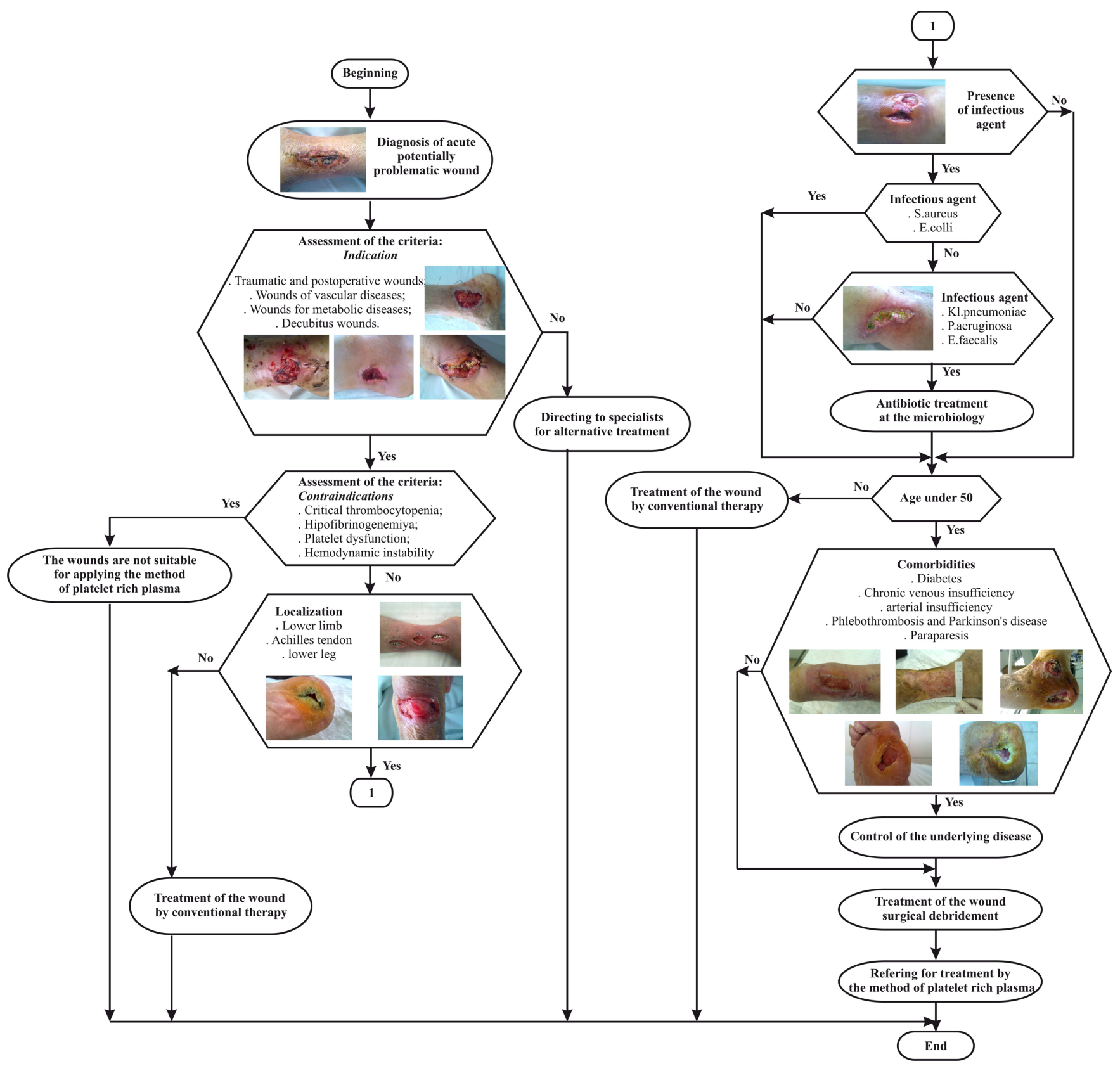


Fig. 2. Algorithm for treatment application of platelet-rich plasma on problematic skin wounds

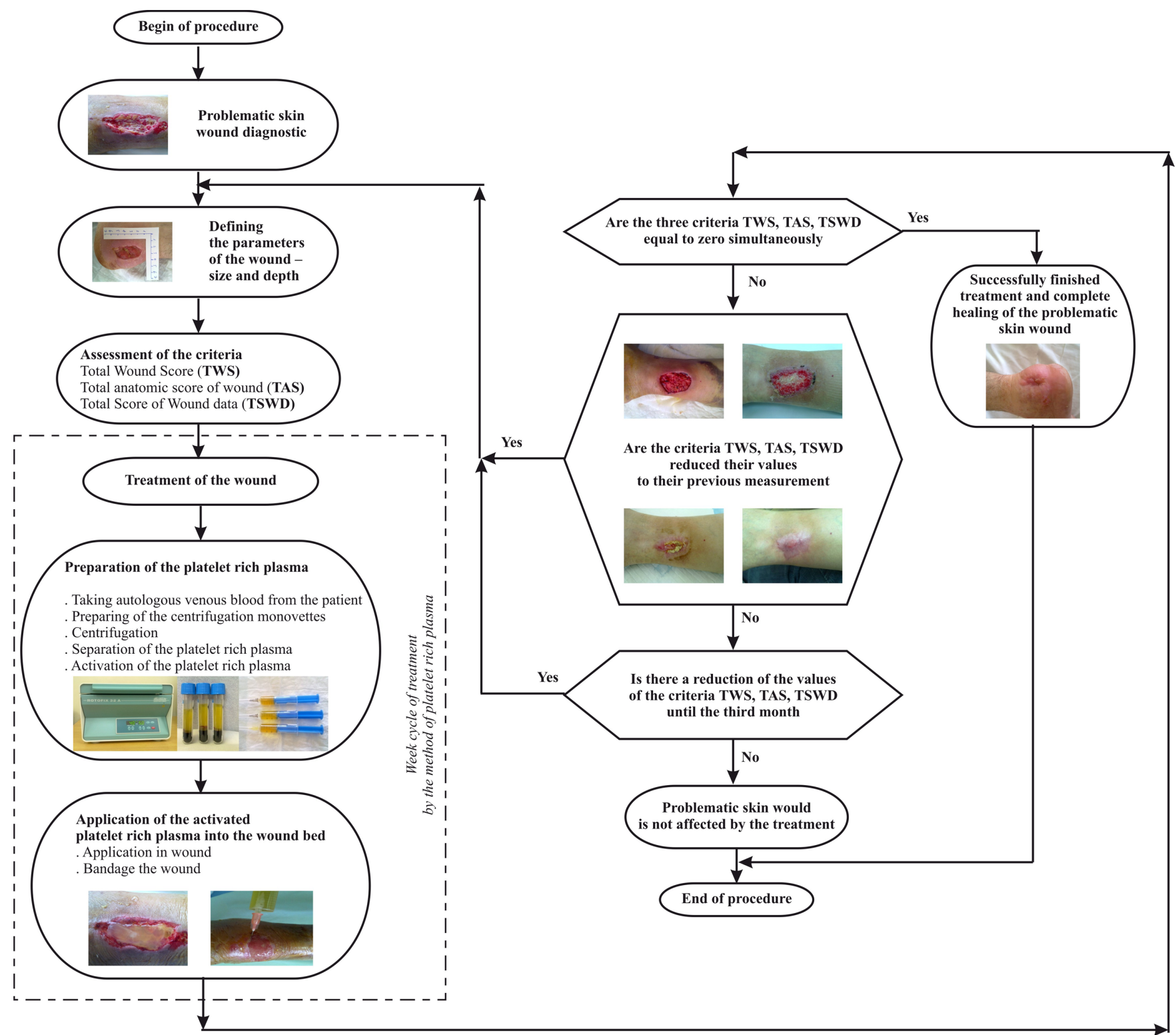

\section{CONCLUSION}

Our own algorithms for prevention and treatment of PSW by PRP increase the percentage of successfully cured wounds. Prevention algorithm of applying platelet-rich plasma ensures that a high percentage of acute skin wounds will not turn into problematic skin wounds. The proposed algorithms for prevention and treatment of problematic skin wounds by applying platelet-rich plasma are an effective and safe way to reduce the uncured complicated skin wounds and ensure the subsequent normal life of patients. They also ensure more predictable skin healing.

\section{Acknowledgements:}

The article reflects the results of the work on the project No. 2020-FOZZG-02 "Development of algorithms for diagnosis and treatment of problematic skin wounds by the method of enriched platelet rich plasma", funded by the Research Fund of the University of Ruse. 


\section{REFERENCES:}

1. Papanas N, Maltezos E. Growth factors in the treatment of diabetic foot ulcers: new technologies, any promises? Int J Low Extrem Wounds. 2007 Mar;6(1):37-53. [PubMed]

2. Clark M. Just the Facts. Number 1. Pressure ulcers. Welsh Wound Network. 2010. [Internet]

3. Hristov V. [Over 100000 Bulgarians suffer from wounds that do not heal!] blitz.bg. 23 January 2014. [in Bulgarian] [Internet]

4. Shoham Y, Krieger Y, Silberstein E, Bogdanov-BerezovskyA, Regev E, Weissman O, et al. Negatively charged microspheres for the treatment of problematic wounds \& patients. EWMA. 2014; poster 235.

5. Sokolov T, Manukova A, Karakoleva S, Valentinov B, Petrova N. Analysis of the results of applying the method Platelet-rich plasma (PRP) for the treatment of problematic skin wounds. J of IMAB. 2017 Jan-Mar; 23(1):1460-1465. [Crossref]

6. Lindholm C, Searle R. Wound management for the 21 st century: combining effectiveness and efficiency. Int Wound J. 2016 Jul;13 Suppl 2:5-15. [PubMed]

7. Crovetti G, Martinelli G, Issi M, Barone M, Guizzardi M, Campanati B, et al. Platelet gel for healing cutaneous chronic wounds. Transfus Apher Sci. 2004 Apr;30(2):145-51. [PubMed]

8. Gottrup F, Jørgensen B. Maggot debridement: an alternative method for debridement. Eplasty. 2011; 11:e33. [PubMed]
9. Cugar R. Foreword I. In: Platelet-Rich Plasma. Regenerative Medicine: Sports Medicine, Orthopedic, and Recovery of Musculoskeletal Injuries. Editors: Lana JFSD, Santana MHA, Belangero WD, Luzo ACM. Springer-Verlag Berlin Heidelberg. 2014. p.5-6 [Crossref]

10. Smith R, Gassmann C, Campbell M. Platelet-rich plasma: properties and clinical applications. JLGH. 2007;2(2):72-78.

11. Cancela AM, Lana JF, Annichino-Bizzachi JM, Belangero WD, Luzo ACM. Use of Platelet-Rich Plasma (PRP) in Treating Chronic Wounds. In: Lana JF, Shimojo AA, Belangero WD, Malheiros Luzo A. (eds.), Platelet-Rich Plasma. Lecture Notes in Bioengineering. SpringerVerlag Berlin, Heidelberg. 2014. p.281-288. [Crossref]

12. Lazarov B, Karakoleva S. Using GAS syntax for education in Mathematics. In: Higher education in Bulgaria and the Europe 2020 strategy, International business school, Botevgrad; 2011. p.914-921.

13. Albanese A, Licata ME, Polizzi B, Campisi G. Platelet-rich plasma (PRP) in dental and oral surgery: from the wound healing to bone regeneration. Immun Ageing. 2013 Jun 13; 10(1):23. [PubMed]

14. Sarvajnamurthy S, Suryanarayan S, Budamakuntala L, Suresh D. Autologous Platelet Rich Plasma in Chronic Venous Ulcers: Study of 17 Cases. J Cutan Aesthet Surg. 2013
Apr;6(2):97-9. [PubMed]

15. Picard F, Hersant B, Bosc R, Meningaud J-P. Should we use plateletrich plasma as an adjunct therapy to treat "acute wounds," "burns," and "laser therapies": A review and a proposal of a quality criteria checklist for further studies. Wound Repair Regen. 2015 Mar-Apr;23(2):163-70. [PubMed]

16. Martin P, Nunan R. Cellular and molecular mechanisms of repair in acute and chronic wound healing. $\mathrm{Br}$ J Dermatol. 2015 Aug;173:370-378. [PubMed]

17. Vandenkerkhof EG, Hopman WM, Carley ME, Kuhnke JL, Harrison MB. Leg ulcer nursing care in the community: a prospective cohort study of the symptom of pain. BMC Nurs. 2013 Feb 6;12:3. [PubMed]

18. Anitua E, Alonso R, Girbau C, Aguirre JJ, Muruzabal F, Orive G. Antibacterial effect of plasma rich in growth factors (PRGF-Endoret) against Staphylococcus aureus and Staphylococcus epidermidis strains. Clin Exp Dermatol. 2012 Aug;37(6):652-7. [PubMed]

19. Tran TD-X, Le PT-B, Pham PV. Diabetic foot ulcer treatment by activated platelet rich plasma: a clinical study. Biomed Res Ther. 2014;1(2):3742.

20. Frykberg RG, Banks J. Challenges in the Treatment of Chronic Wounds. Adv Wound Care (New Rochelle). 2015 Sep 1;4(9):560-582. [PubMed] [Crossref]

Please cite this article as: Sokolov T, Manukova A, Kovachev V, Mancho Kovachev M. Treatment of Problematic Skin Wounds Based on the Platelet-Rich Plasma Method. Our own Algorithms for Application. J of IMAB. 2020 OctDec;26(4):3436-3442. DOI: https://doi.org/10.5272/jimab.2020264.3436

\author{
Address for correspondence: \\ Dr Tsvetan V. Sokolov, \\ Clinic of Orthopedics and Traumatology, UMHAT Kanev, Ruse, Bulgaria \\ 2, Nezavisimost str., 7002 Ruse, Bulgaria. \\ E-mail: sokolovi2001@abv.bg
}

